\title{
Single-chain self-folding in an amphiphilic copolymer: an integrated experimental and computational study
}

\author{
Elisa Guazzelli ${ }^{1}$, Elisa Martinelli ${ }^{1,2}$, Giancarlo Galli ${ }^{1,2 *}$, Lorenzo Cupellini ${ }^{1}$, Sandro Jurinovich ${ }^{1}$, \\ Benedetta Mennucci ${ }^{1 *}$
}

${ }^{1}$ Dipartimento di Chimica e Chimica Industriale, Università di Pisa, 56124 Pisa, Italy

${ }^{2}$ Consorzio INSTM, UdR Pisa, 56124 Pisa, Italy

E-mail: giancarlo.galli@unipi.it, benedetta.mennucci@unipi.it

Supplementary Data available: Details on synthesis of copolymer and molecular dynamics simulations.

Keywords: amphiphilic polymer, single-chain folding, self-assembly, molecular dynamics, prolate nanoassembly

\begin{abstract}
An amphiphilic random copolymer of hydrophilic of poly(ethylene glycol) methyl ether methacrylate with hydrophobic perfluorohexylethyl acrylate, PEGMA77-co-FA23, was synthesized by ATRP and used to investigate self-assembling into nanostructures in water and chloroform solutions, both experimentally and computationally. The dynamic light scattering measurements on water solutions of the copolymer at room temperature evidenced the presence of nanoassemblies with hydrodynamic diameter $D_{\mathrm{h}}=4 \pm 1 \mathrm{~nm}$. The behavior of fluorescence emission intensity of water solutions with added ethidium bromide suggested confinement of the molecular rotor within a hydrophobic environment of the copolymer. Moreover, these nanoassemblies were thermoresponsive and reversibly collapsed into much larger, multi-chain aggregates with $D_{\mathrm{h}}=390 \pm 20 \mathrm{~nm}$ at a critical temperature of $55{ }^{\circ} \mathrm{C}\left(5 \mathrm{mg} \mathrm{mL}^{-1}\right)$. Molecular dynamics simulations revealed the formation of single-chain, prolate globular nanoassemblies with a structural variability in water solution at room temperature. The evolutions of the simulated radius of gyration $\left(R_{\mathrm{g}}\right)$, asphericity, prolateness and solvent-accessible surface area were analyzed along the folding trajectories. Thus, selffolding appeared to result from the interplay between hydrophobic interactions and structural constraints which leads to rather complex nanostructures $\left(R_{\mathrm{g}}=20-25 \AA\right.$, 200 ns simulation). By contrast, folding in much more open polymer conformations $\left(R_{\mathrm{g}}=30-40 \AA\right)$ was predicted for chloroform solutions.
\end{abstract}

\section{Introduction}

The concept behind the fast developing field of single-chain nanoparticles (SCNPs) is straightforward in principle and clearly inspired by the precise and efficient folding of natural macromolecules, that can provide complex functions related to their three-dimensional arrangement.[1-4] The preparation, characterization and practical application of such artificial single-chain nanostructures are less obvious, nevertheless the benefits would be uncountable for example in catalysis, sensors, vectors for contrast agents, drug delivery carriers, and in nanotechnology at large.[5] Several strategies have been pursued to collapse and stabilize single polymer chains into functional, soft nanoobjects, involving the formation of (reversible) covalent bonds and non-covalent intrachain interactions.[4,6-14] 
The self-assembly of neutral amphiphilic random copolymers represents one straightforward approach to generate dynamic, reversible, stimulus-responsive SCNPs in water, via hydrophobic intramolecular interactions established between water-insoluble portions of the copolymer.[15] These special SCNPs are sometimes referred to as unimer micelles, in analogy with the intermolecular micelles formed by common amphiphiles. Repeat units with different degree of hydrophobicity have been reported, such as those comprised of alkyl [15], fluoroalkyl [16-18] and siloxane [19] chains. In particular, the inclusion of low surface energy fluoroalkyl side chains in a polymer structure is known to drastically enhance the hydrophobic and lipophobic nature of the entire system, which results in a strong capability to self-assemble in solution, in bulk and at the surface of thin films.[20-24] Hydrophobic monomers are randomly copolymerized with a large excess of hydrophilic monomers, mainly poly(ethylene glycol) based monomers such as methacrylate (PEGMA) [15-19] or acrylamide (PEGAAm).[25] PEG-based copolymers have the advantage to be soluble in both water and organic solvents, with effective steric stabilization of the formed SCNPs even at high concentrations in water.[15] Moreover, they inherit a lower critical solution temperature-type (LCST) behavior in water solution, typical of the corresponding PEGMA homopolymers, leading to thermoresponsive systems.[26-28]

Valuable information to guide the development of single-chain technology would come from theoretical and computational approaches, especially in the delicate matter of unraveling morphological and conformational aspects to be correlated with the manifold collection of experimental data.[29,30] Molecular dynamics (MD) and Monte Carlo (MC) simulations mostly using coarse grained models have been used to investigate soft nanoparticles, mainly formed by intramolecular covalent cross-linking [31-33] or hydrogen bonding supramolecular motifs.[34] Owing to the large reduction in the number of explicit degrees of freedom allowed by these techniques, longer time windows become available as well as longer chains can be investigated. From these analyses and from the comparison of a large number of experimental data, it appears that chain compaction occurs locally, independent of the type of intramolecular interactions, while the overall structure maintains an open and swollen conformation resembling that of intrinsically disordered proteins.[29,30] The physical reason for this lack of compactness has been related to the fact that self-avoiding conformations of the linear precursors in the good solvent conditions of the synthesis make the formation of long loops statistically infrequent. As a result, cross-linking events are found only between groups separated by short contour distances and a large-scale compaction cannot be achieved. Simulations have also been used to develop different protocols allowing bonding between groups separated by long contour distances. Some of these strategies are based on the use of bad solvent conditions for the reactive groups which leads to a collapsed conformation of the precursor.[35,36] While the solvent affinity displayed some effects on the compaction of covalently crosslinked SCNPs, it plays a critical role in the spontaneous formation of single-chain folded nanostructures (unimer micelles). In this case, in fact, the driving forces of the folding process and the stabilization of the resulting nanoassemblies are those generated exclusively from the hydrophobic and hydrophilic interactions of different components of the copolymer with water, which acts as a selective solvent. It was assumed that such selffolded assemblies adopt in water a closed and compact, spherical nanostructure consisting of a highly hydrophobic core and a highly hydrophilic shell,[15,17] but solid experimental and computational support to their actual shape and morphology is still lacking.

Herein, an ATRP random amphiphilic copolymer from hydrophilic poly(ethylene glycol) methyl ether methacrylate (PEGMA) and hydrophobic/lipophobic perfluorohexylethyl acrylate (FA) was used to build model nanoassemblies by single-chain self-folding of the copolymer in water, driven by hydrophobic interaction between the 
perfluorinated side chains.[16-18] Dynamic light scattering and fluorescence emission spectroscopy with ethidium bromide fluorescent probe were used to characterize the self-assembly of the copolymer in water, including its temperature-dependent mode of aggregation. In order to achieve a detailed understanding of the folding mechanism, fully atomistic MD simulations were carried out on the copolymer in water and in chloroform, chosen as selective and non-selective solvents for the amphiphilic copolymer, respectively. The resulting trajectories suggested strongly different behaviors in the two solvents. In particular, only in water, the copolymer single-chains fold into prolate globular nanoassemblies that present a structural heterogeneity.

\section{Experimental}

\subsection{Materials}

Anisole (Sigma Aldrich) was vacuum distilled over sodium. CuBr (Sigma Aldrich) was extracted with glacial acetic acid then washed with diethyl ether, dried and stored under nitrogen. $N, N, N^{\prime}, N^{\prime \prime}, N^{\prime \prime}$ Pentamethyldiethylenetriamine (PMDETA, Sigma Aldrich), and ethyl $\alpha$-bromophenylacetate (EBPA, Sigma Aldrich) were freshly distilled before use. Perfluorohexylethyl acrylate (FA, Fluoryx), and poly(ethylene glycol) methyl ether methacrylate (PEGMA, $M_{\mathrm{n}}=300 \mathrm{~g} \mathrm{~mol}^{-1}, \oslash=1.2$, Sigma Aldrich) were filtered through basic alumina to remove inhibitors. Ethidium bromide (EtBr, purity 99\%, Sigma) solutions were prepared by dissolving weighed amounts in water and their molar concentration was verified spectrophotometrically $\left(\varepsilon=5600 \mathrm{M}^{-1} \mathrm{~cm}^{-1}\right.$ at $\left.\lambda_{\max }=480 \mathrm{~nm}\right)$.[37]

\subsection{Characterization}

\subsubsection{Nuclear magnetic resonance (NMR)}

${ }^{1} \mathrm{H}$ NMR and ${ }^{19} \mathrm{~F}$ NMR solution spectra were recorded with a Bruker Avance DRX 400 spectrometer. The ${ }^{1} \mathrm{H}$ NMR spectra in various solvents were used to evaluate the $M_{\mathrm{n}}$ of the copolymer.

\subsubsection{Size exclusion chromatography (SEC)}

Values of number and weight average molecular weights of the copolymer $\left(M_{\mathrm{w}}, M_{\mathrm{n}}\right)$, Mark-Houwink slope $(\alpha)$ and increment of refractive index $(\mathrm{d} n / \mathrm{d} C)$ were determined by SEC measurements with a SEC Max Malvern instrument equipped with a Viscotek TDA 305 apparatus with three detectors (refractive index, detection angle at $90^{\circ}$ and low angle $\left(7^{\circ}\right)$ laser light scattering, viscometer). Copolymer solutions in various solvents were used at $40-60{ }^{\circ} \mathrm{C}$ : flux: $0.8 \mathrm{~mL} \mathrm{~min}{ }^{-1}$; injection volume: $0.100 \mu \mathrm{L}$; columns: Phenogel $10^{6}$ and $10^{3}$ with a Phenogel pre-column; triple detection (polystyrene standard, $M_{\mathrm{n}}=101,252 \mathrm{~g} \mathrm{~mol}^{-1}, M_{\mathrm{w}}=104,959 \mathrm{~g} \mathrm{~mol}^{-1}$ ) and universal calibration (polystyrene standard, $M_{\mathrm{n}}=935-3,000,000 \mathrm{~g} \mathrm{~mol}^{-1}$ ).

The values of $M_{\mathrm{w}}, M_{\mathrm{n}}$ and dispersity $(\Theta)$ of samples from polymerization solutions for kinetics experiments were evaluated by SEC with a Jasco PU-2089Plus liquid chromatograph equipped with two PL gel $5 \mu \mathrm{m}$ mixed-D columns, a Jasco RI-2031Plus refractive index detector and a Jasco UV-2077Plus UV/vis detector. Poly(methyl methacrylate) standards $\left(M_{\mathrm{n}}=1160-124,300 \mathrm{~g} \mathrm{~mol}^{-1}\right)$ were used for calibration.

\subsubsection{Dynamic light scattering (DLS)}

DLS measurements on polymer solutions were taken with a Beckman Coulter Delsa Nano C particle analyzer (detection angle $=166.22^{\circ}$ ). Intensity, volume and number distribution were obtained from the signal autocorrelation function through CONTIN analysis in the instrument software. Hydrodynamic diameters were evaluated from intensity 
size distributions and averaged over at least 5 measurements. Samples $\left(5 \mathrm{mg} \mathrm{mL} \mathbf{m}^{-1}\right.$ copolymer solutions) were prepared in filtered solvents $(0.2 \mu \mathrm{m}$ CA or PTFE filters) of the highest purity available to avoid external contamination. All the solutions were analyzed at $25^{\circ} \mathrm{C}$ and at temperatures varied in steps of $\sim{ }^{\circ} \mathrm{C}$, in both heating and cooling cycles.

\subsubsection{Fluorescence emission spectroscopy}

A Perkin Elmer LS55 spectrofluorometer was used to record fluorescence spectra, with a temperature control of $\pm 0.1^{\circ} \mathrm{C}$.

\subsection{Synthesis}

\subsubsection{Synthesis of copolymer}

PEGMA (4.59 mL, $16.07 \mathrm{mmol})$, FA (1.679 g, $4.02 \mathrm{mmol})$, PMDETA (41.9 $\mu \mathrm{L}, 0.20 \mathrm{mmol})$, EBPA (35.2 $\mu \mathrm{L}, 0.20$ $\mathrm{mmol}$ ) and anisole $(3.25 \mathrm{~mL})$ were degassed in a Schlenk tube with three freeze-pump-thaw cycles. Then, CuBr (28.8 $\mathrm{mg}, 0.20 \mathrm{mmol}$ ) was added and three more freeze-pump-thaw cycles were performed before the polymerization was started at $70{ }^{\circ} \mathrm{C}$ under nitrogen atmosphere. Periodically, aliquots of the reaction mixture were withdrawn for kinetics and molar mass analysis. After $24 \mathrm{~h}$, the reaction was stopped by exposure to air and quenching to $0{ }^{\circ} \mathrm{C}$. The crude product was filtered several times through basic alumina to remove catalyst and repeatedly precipitated from chloroform solutions into $n$-hexane to isolate copolymer (final yield 54\%).

${ }^{1} \mathrm{H}$ NMR [acetone- $d_{6}$ ] $: \delta(\mathrm{ppm})=7.2-7.4\left(0.5 \mathrm{H}\right.$, aromatic), 4.1-4.5 $\left(21.0 \mathrm{H}, \mathrm{COOCH}_{2}\right), 3.4-3.8\left(139.5 \mathrm{H}_{,} \mathrm{OCH}_{2} \mathrm{CH}_{2}\right), 3.3$ $\left(24.0 \mathrm{H}, \mathrm{OCH}_{3}\right), 2.7-2.9\left(\mathrm{CH}_{2} \mathrm{CF}_{2}+\mathrm{H}_{2} \mathrm{O}^{* *}\right), 1.5-2.3\left(\mathrm{CH}_{2}, \mathrm{CH}+\right.$ acetone- $\left.d_{6}^{*}\right), 0.8-1.5\left(24.9 \mathrm{H}, \mathrm{CH}_{3}\right)(* *$ and *: water and solvent impurities) (see Supplementary Data, Figure S1).

${ }^{19} \mathrm{~F} \mathrm{NMR}$ [acetone- $\left.d_{6}, \mathrm{CF}_{3} \mathrm{COOH}\right]: \delta(\mathrm{ppm})=-5.6\left(3.0 \mathrm{~F}, \mathrm{CF}_{3}\right),-38.5\left(2.0 \mathrm{~F}, \mathrm{CF}_{2} \mathrm{CH}_{2}\right),-46$ to $-48\left(6.1 \mathrm{~F}, \mathrm{CF}_{2}\right),-51(2.0 \mathrm{~F}$, $\mathrm{CF}_{2} \mathrm{CF}_{3}$ ) (Figure $\mathrm{S} 2$ ).

\subsection{Computational details}

\subsubsection{Force field and model structures}

The amphiphilic copolymer was composed of PEGMA and FA counits capped with a methyl group at both ends. We generated four different model 104-mer random sequences (RA-RD) with an approximate number of 80 PEGMA/24 FA counits by randomly selecting the PEGMA or FA counits. The resulting structures had the following compositions: RA (76 PEGMA/28 FA), RB (87 PEGMA/17 FA), RC (75 PEGMA/29 FA), and RD (79 PEGMA/25 FA). Moreover, we generated one blocky sequence (EQB) with 80 PEGMA counits and 24 FA counits and one PEGMA homopolymer (all-HYD). Parameters for the polymer were taken from the General Amber Force Field (GAFF) [38] and partial charges were derived following the standard RESP procedure.[39] Parameters for the solvents were modeled or taken from the literature.[40,41] Further details on the preparation of the system are presented in the Supplementary Data. 


\subsubsection{Folding simulation}

The initial structure was put into a rectangular box of water molecules. The four different model structures were also solvated in chloroform. The dimensions of the box were chosen so that the edges of the box were at a minimum distance of $20 \AA$ from the polymer atoms. The solvated system was heated up to $300 \mathrm{~K}$ for $100 \mathrm{ps}$, followed by a $200 \mathrm{~ns}$ of production run. All the MD simulations were performed in a NPT ensemble using a Langevin thermostat with a damping constant of $1 \mathrm{ps}^{-1}$ and the isotropic Monte Carlo barostat as implemented in Amber 14.[42] The integration time step was set to $2 \mathrm{fs}$ in combination with the SHAKE algorithm for hydrogen atoms.

\subsubsection{Analysis of trajectories}

Different parameters were analyzed along the folding trajectories. We extracted the gyration tensor $(G)$, defined as:

$$
\mathbf{G}_{\mathrm{ij}}=\frac{1}{N} \sum_{n=1}^{N} \mathbf{r}_{n, i} \mathbf{r}_{n, j} \quad i, j=x, y, z
$$

where $i$ and $j$ are two cartesian components, the sum runs over all the heavy atoms of the polymer, and $\mathbf{r}_{n}$ is the position of the $n$-th atom with respect to the geometric center of the polymer. Note that the positions are not massweighted. To describe the dimensions of the polymer, we used the radius of gyration, defined as $R_{\mathrm{g}}=\sqrt{\operatorname{tr}(\mathbf{G})}$. Since the radius of gyration only estimates the compactness of the macromolecule, it is useful to define additional shape descriptors from the principal gyration moments, i.e. the eigenvalues of the gyration tensor $G_{X}<G_{Y}<G_{Z}$, associated with the principal axes $X, Y$, and $Z$.

The asphericity parameter $\alpha$ and the prolateness parameter $p$ are defined $\mathrm{as}^{31,32}$ :

$$
\begin{aligned}
& \alpha=\frac{\left(G_{X}-G_{Y}\right)^{2}+\left(G_{X}-G_{Z}\right)^{2}+\left(G_{Y}-G_{Z}\right)^{2}}{2\left(G_{X}+G_{Y}+G_{Z}\right)} \\
& p=\frac{\left(3 G_{X}-R_{g}^{2}\right)\left(3 G_{Y}-R_{g}^{2}\right)\left(3 G_{Z}-R_{g}^{2}\right)}{2\left(G_{X}^{2}+G_{Y}^{2}+G_{Z}^{2}-G_{X} G_{Y}-G_{X} G_{Z}-G_{Y} G_{Z}\right)^{3 / 2}}
\end{aligned}
$$

The asphericity $\alpha$ is always positive and $\leq 1$, and quantifies the deviation from a spherical shape. For a spherically symmetrical object, $\alpha=0$, whereas for a perfectly linear object $\alpha=1$. The prolateness $p$ has values between -1 and 1 , and is positive (negative) for a prolate (oblate) object. In particular, $p=1$ when $G_{X}=G_{Y}<G_{Z}$, and $p=-1$ when $G_{X}<G_{Y}=$ $G_{z}$. These two descriptors can thus be used to classify the shape of the polymer, giving complementary information to $R_{\mathrm{g}}$.

In order to assess the role of hydrophobic interactions, we computed the solvent-accessible surface area (SASA) [43] of the polymer using the SURF program.[44] The SASA was partitioned into individual contributions from PEGMA and FA counits (SASA $A_{P E G M A}$ and $\left.S A S A_{F A}\right)$ and analyzed along the trajectory by defining the time-dependent fraction $X_{P E G M A / F A}(t)$ as:

$$
X_{\text {PEGMAFA }}(t)=\frac{S A S A_{P E G M A \mid F A}(t)}{S A S A_{P E G M A \mid F A}(0)}
$$




\section{Results and discussion}

\subsection{Synthesis of random copolymer}

An amphiphilic random copolymer from PEGMA and FA was synthesized via atom transfer radical polymerization (ATRP) (Scheme 1), using EBPA as initiator, CuBr as catalyst, PMDETA as ligand (1:1:1 molar ratios) and anisole $\left(0.5 \mathrm{~mL} \mathrm{~g}^{-1}\right.$ of monomers) as solvent at $70{ }^{\circ} \mathrm{C}$. The resulting copolymer was named PEGMA77-Co-FA23, according to the mole percentage contents of the respective repeat counits. The evaluated monomer reactivity ratios, $r_{\mathrm{PEGMA}}=1.43$ and $r_{\mathrm{FA}}=0.88$ (AIBN initiation, $5 \mathrm{~min}$ reaction time, Kelen-Tudos method), suggested a random insertion of counits within the copolymer backbone although a little gradient in composition was produced due to the drift in feed composition occurring during the reaction.
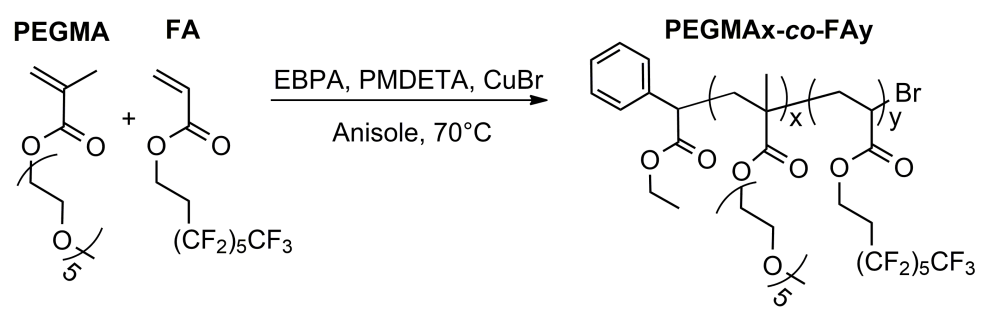

Scheme 1. Synthesis of the amphiphilic random copolymer PEGMA77-co-FA23 via ATRP $(x=77, y=23$ mol\%).

Key features of the reversible-deactivation reaction of the ATRP were verified by monitoring the kinetics of copolymer formation by ${ }^{1} \mathrm{H}$ NMR and SEC analyses. Figure 1 a shows the kinetics plots of $\ln \left([M]_{0} /[M]\right)$ as a function of reaction time for PEGMA and FA. Both plots have a linear trend up to $75 \%$ total monomer conversion, indicating that the polymerization proceeded with a first order kinetics. A deviation from linearity was observed at higher conversions (Figure S3), denoting the incidence of termination reactions. Chain transfer reactions to ethylene glycol side chains may also occur. $[45,46]$ The values of the overall number average degree of polymerization $D P_{\mathrm{n}}$ determined were consistent with the theoretical values expected for an ATRP process (Figure 1b) calculated as:

$$
D P_{\mathrm{n}}=\frac{[M]_{0}}{[I]_{0}} p
$$

where $p$ is the monomer conversion and $[M]_{0}$ and $[I]_{0}$ are the initial molar concentrations of both monomers and initiator, respectively. Narrow dispersity indexes, $\oslash \leq 1.35$, were evaluated by SEC (Figure 1c). The ${ }^{19} \mathrm{~F}$ NMR spectra (Figure S2) confirmed the inclusion of fluorinated counits in the copolymer. 


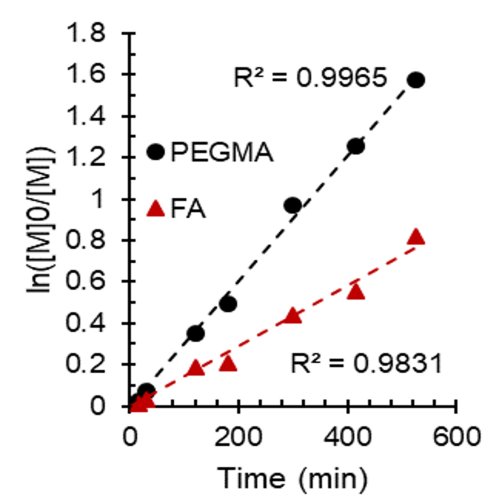

(a)

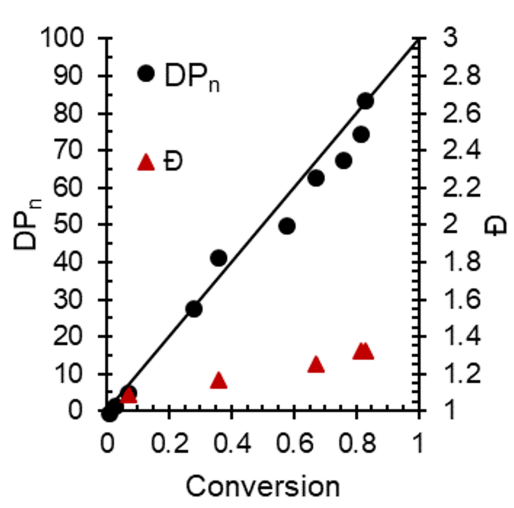

(b)

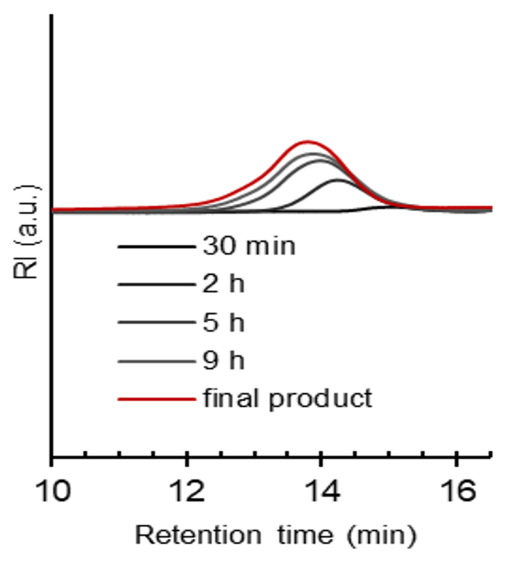

(c)

Fig. 1. (a) Kinetics plots, (b) overall degree of polymerization $\left(D P_{n}\right.$, by ${ }^{1} \mathrm{H} N M R$ in acetone) and dispersity index $(\Theta$, by $\operatorname{SEC}$ in chloroform) vs conversion, and (c) evolution of the SEC curves of the copolymer during the polymerization time.

The average molecular weights were investigated by SEC experiments with triple detection and/or universal calibration in various solvents. Values of $M_{\mathrm{n}}=25000 \mathrm{~g} \mathrm{~mol}^{-1}$ and $M_{\mathrm{w}}=34500 \mathrm{~g} \mathrm{~mol}^{-1}(\Theta=1.38)$ were determined for solutions (with both triple detection and universal calibration) in tetrahydrofuran $(\alpha=0.38)$. Triple detection was not possible on solutions in chloroform or dimethylformamide (10 $\mathrm{mM} \mathrm{LiBr})$, owing to a very weak scattering response at the detecting angles; universal calibration provided values of $M_{\mathrm{n}}=15500 \mathrm{~g} \mathrm{~mol}^{-1}$ and $M_{\mathrm{w}}=21000 \mathrm{~g} \mathrm{~mol}^{-1}(\theta=1.35)$ for the former solutions $(\alpha=0.46)$ and values of $M_{\mathrm{n}}=25000 \mathrm{~g} \mathrm{~mol}^{-1}$ and $M_{\mathrm{w}}=34500 \mathrm{~g} \mathrm{~mol}^{-1}(\theta=1.38)$ for the latter $(\alpha=0.38)$. All such values of Mark-Houwink slope suggest the existence of a random coil polymer chain in these solutions, with chloroform being a better (theta-like) solvent. Meaningful values of molecular weights and viscometry parameters $(\alpha, \log K)$ could not be obtained for the copolymer solutions in water $\left(0.02 \mathrm{wt} \% \mathrm{NaN}_{3}\right)$ or water/methanol (90/10 v/v) by either SEC method. This was possibly due to a worse copolymer solvation in these SEC conditions, with a very low increment of refractive index in both solutions $\left(\mathrm{d} n / \mathrm{d} C=0.004 \mathrm{~mL} \mathrm{~g}^{-1}\right)$. Nonetheless, values of $M_{\mathrm{n}}$ of $37200 \mathrm{~g}$ $\mathrm{mol}^{-1}$ in water and $34000 \mathrm{~g} \mathrm{~mol}^{-1}$ in both chloroform $\left(M_{\mathrm{n}, \text { chloroform }} / M_{\mathrm{n} \text {, water }} \approx 0.9\right)$ and acetone solutions were evaluated by ${ }^{1} \mathrm{H}$ NMR (Figure S1). Therefore, the copolymer formed unimolecular nanostructures in solution (see also below). Accordingly, average numbers of repeat counits in the copolymer were 80 for PEGMA and 24 for FA.

\subsection{Dynamic light scattering of water solutions}

DLS measurements of water solutions of the amphiphilic copolymer were performed to directly assess its ability to self-assemble in water at room temperature and as a function of temperature. Figure $2 a$ shows the volume size distribution for copolymer clear solution in water at $25{ }^{\circ} \mathrm{C}$ that presented a predominant population of nanostructures with hydrodynamic diameter $D_{\mathrm{h}}=4 \pm 1 \mathrm{~nm}$. A very minor population of nanostructures with $D_{\mathrm{h}}=170 \pm$ $7 \mathrm{~nm}$ was also detected. $D_{\mathrm{h}}$ value was in agreement with the existence in water solution of unimolecular nanoassemblies, identified as unimer micelles of single-chain folded copolymer.[16-18] These were formed by the self-aggregation of the hydrophobic FA side chains in an inner compartment, whereas the hydrophilic PEGMA side chains were preferentially exposed to contact with water in a outer shell. 


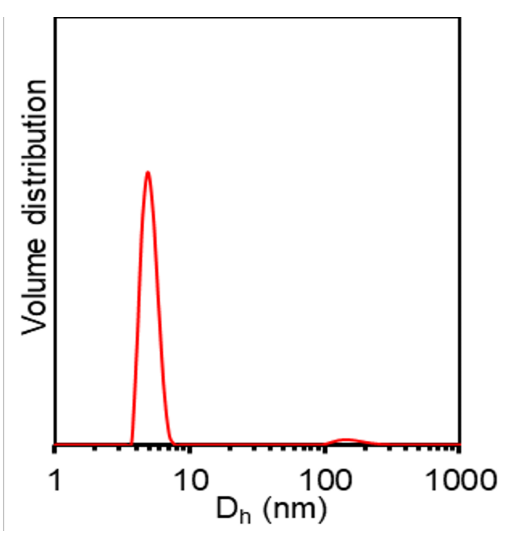

(a)

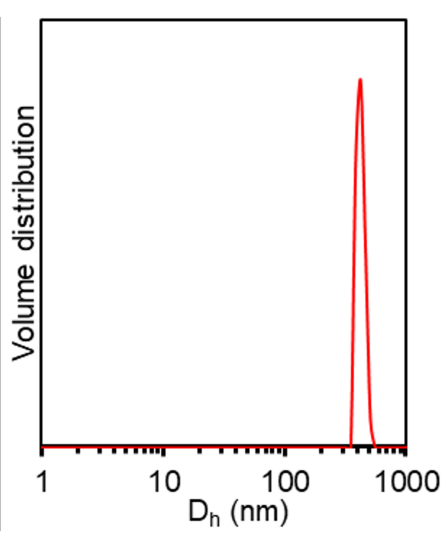

(b)

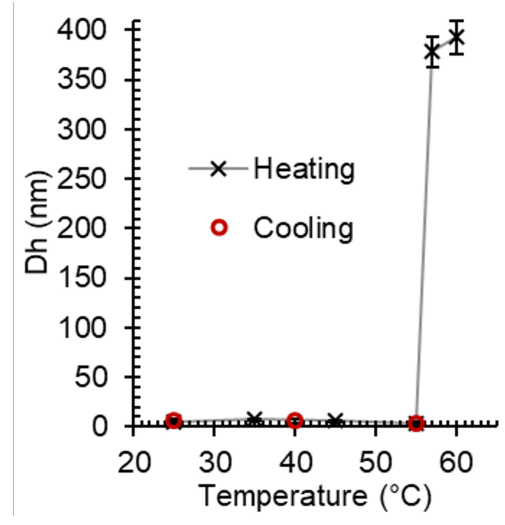

(c)

Fig. 2. DLS volume size distributions for copolymer solutions in water $\left(5 \mathrm{mg} \mathrm{mL}^{-1}\right)$ at (a) $25^{\circ} \mathrm{C}$, (b) $60{ }^{\circ} \mathrm{C}$, and (c) variation of the hydrodynamic diameter $\left(D_{\mathrm{h}}\right)$ as a function of temperature.

DLS measurements on water solutions $\left(5 \mathrm{mg} \mathrm{mL}^{-1}\right)$ as a function of temperature revealed that above a critical temperature $\left(T_{\mathrm{c}}=55^{\circ} \mathrm{C}\right)$, the stable nanoassemblies collapsed into much larger multi-chain aggregates with $D_{\mathrm{h}}=390 \pm$ $20 \mathrm{~nm}$ with a very narrow size distribution (PDI $=0.006$ ) (Figure $2 \mathrm{~b}$ ), characteristic of a lower critical solution temperature (LCST) behavior. DLS measurements confirmed that this phenomenon was reversible with no temperature hysteresis on cooling (Figure $2 \mathrm{c}$ ). Values of $T_{\mathrm{c}}$ in the range $66-76{ }^{\circ} \mathrm{C}$ were reported for other polymers based on PEGMA $\left(M_{n}=300 \mathrm{~g} \mathrm{~mol}^{-1}\right) \cdot[27,28]$ The amphiphilic random copolymer of this work showed a similar thermoresponsive behavior, by which hydrogen bonds between water and hydrophilic oxyethylene side chains of single-chain nanoassemblies are broken at $T_{\mathrm{c}}$ and the chains collapse into larger multi-chain aggregates. However, the marked hydrophobic nature of the FA counits led to significantly lower $T_{c}^{\prime}$ 's than those of the PEGMA homopolymer. DLS measurements carried out on chloroform solutions of the copolymer revealed the formation of scattering nanostructures with hydrodynamic diameter $D_{\mathrm{h}}=5 \pm 1 \mathrm{~nm}$. Thus, the copolymer chains were folded in more open conformations in this solvent.

\subsection{Fluorescence emission of water solutions with ethidium bromide}

Ethidium bromide (EtBr) is a weakly fluorescent molecule in water, known for its ability of tightly intercalating into DNA, thus extensively enhancing its fluorescence intensity.[47] Similarly, when added to an aqueous solution of the copolymer, an increase in its fluorescence emission $\left(\lambda_{\mathrm{exc}}=520 \mathrm{~nm}\right)$ was observed, owing to hydrophobic interactions between the aromatic core of $\mathrm{EtBr}$ and the inner compartment of the copolymer nanoassemblies (Figure 3a). In this way, the interaction with solvent molecules was shielded, and fluorescence emission from a locally excited state was enhanced.

Given the inherent thermoresponsive behavior of the amphiphilic copolymer in water, the temperature dependence of the fluorescence emission of the copolymer/EtBr system in water was also examined. As an example, the fluorescence emission intensity at $607 \mathrm{~nm}\left(\lambda_{\mathrm{exc}}=520 \mathrm{~nm}\right)$ of a water solution containing the copolymer (3.13 $\mathrm{mg}$ $\left.\mathrm{mL}^{-1}\right)$ and $\mathrm{EtBr}\left(0.03 \mathrm{mg} \mathrm{mL}^{-1}\right)$ significantly decreased in going from $25^{\circ} \mathrm{C}$ to $65^{\circ} \mathrm{C}$ (Figure $3 \mathrm{~b}$ ). On the other hand, an increase in scattering (at $530 \mathrm{~nm}$ ) due to the formation of larger sub-microaggregates at higher temperatures was evident. These two opposite trends showed a discontinuity at $52{ }^{\circ} \mathrm{C}$ (Figure 3c). Above $52{ }^{\circ} \mathrm{C}$ single-chain folded macromolecules aggregated, consistent with the observations by DLS. On collapsing, the multi-chain aggregates 
expelled solvation water molecules, no longer engaged in hydrogen bonds with the oxyethylene side chains, and simultaneously released $\mathrm{EtBr}$ to the outer environment, as is shown by the significant sudden decrease in its fluorescence emission intensity.

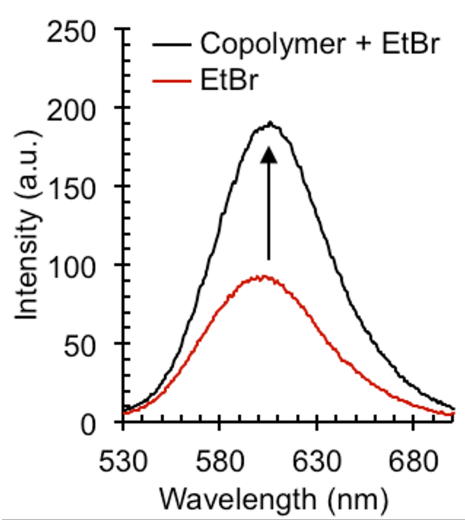

(a)

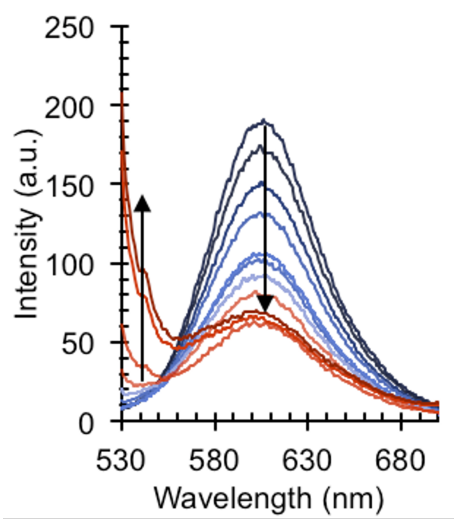

(b)

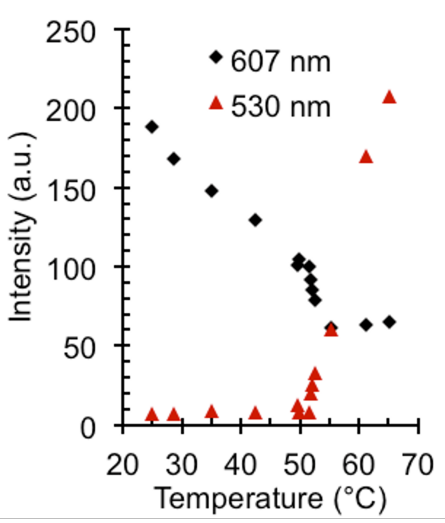

(c)

Fig. 3. (a) Fluorescence emission spectra $\left(\lambda_{\text {exc }}=520 \mathrm{~nm}\right)$ in water of a reference solution of $\operatorname{EtBr}\left(0.03 \mathrm{mg} \mathrm{mL}^{-1}\right)$ and a solution of copolymer $\left(3.13 \mathrm{mg} \mathrm{mL}^{-1}\right)$ containing $\operatorname{EtBr}\left(0.03 \mathrm{mg} \mathrm{mL}^{-1}\right)$. (b) Fluorescence emission spectra $\left(\lambda_{\text {exc }}=520 \mathrm{~nm}\right)$ in water of a solution of copolymer $\left(3.13 \mathrm{mg} \mathrm{mL}\right.$ ) containing $\mathrm{EtBr}\left(0.03 \mathrm{mg} \mathrm{mL}^{-1}\right.$ ) at different temperatures from 25 to $65{ }^{\circ} \mathrm{C}$ (from blue to red, respectively). (c) Fluorescence emission intensity maxima at $607 \mathrm{~nm}$ and scattering intensity at $530 \mathrm{~nm}$ as a function of temperature for a solution of copolymer $\left(3.13 \mathrm{mg} \mathrm{mL}^{-1}\right)$ in water containing $\operatorname{EtBr}\left(0.03 \mathrm{mg} \mathrm{mL}^{-1}\right)$.

Solutions with different concentrations of PEGMA77-co-FA23 exhibited an analogous LCST-type behavior, but with different transition temperatures $T_{\mathrm{c}}$ (Figure 4). These results showed that the multi-chain aggregation and the associated release of EtBr were induced at higher temperatures $\left(74-55^{\circ} \mathrm{C}\right)$ for dilute solutions $\left(0.10-0.63 \mathrm{mg} \mathrm{mL}^{-1}\right)$. Above $2.34 \mathrm{mg} \mathrm{mL}^{-1}$ and up to significantly higher copolymer concentration of $32.85 \mathrm{mg} \mathrm{mL}^{-1}$, the EtBr release was triggered at $53-51{ }^{\circ} \mathrm{C}$. Such temperature values were consistent with those previously found by DLS.

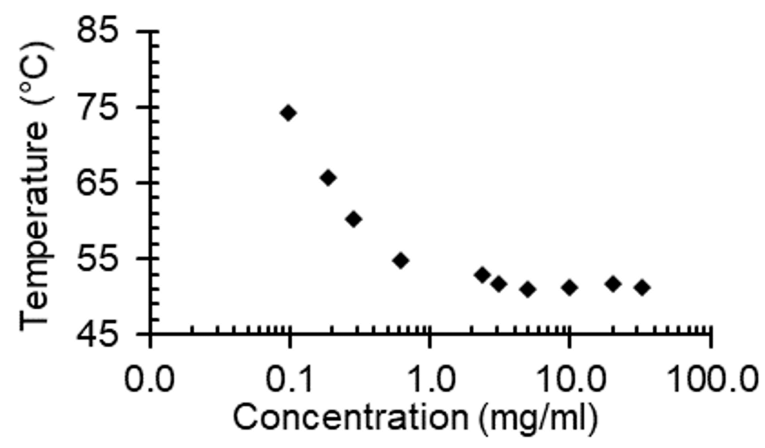

Fig. 4. Critical temperature $T_{\mathrm{c}}$ vs copolymer concentration in water.

It was recently observed that the $\mathrm{EtBr}$ can interact with single-chain folded nanoassemblies derived from similar PEGMA-Co-FA copolymers which were functionalized by incorporating a julolidine-based fluorescent molecular rotor (FMR) as a comonomer.[18] The spectroscopic absorption/emission properties of FMR and EtBr closely matched with each other, and a Förster resonance energy transfer (FRET) process was observed, implying a short range interaction between the FMR donor and the EtBr acceptor. However, despite the proximity within the nanoassembly, $\mathrm{EtBr}$ fluorescence emission was weak, since it was not sheltered enough from the surrounding water molecules. Formation of a more complex and looser structure is supposed for these nanoassemblies compared to the spherical and compact core-shell morphology that has been shown for nanoassemblies of structurally related random PEGMA- 
fluoroalkyl methacrylate (PEGMA-co-FMA) copolymers in water solution.[16,17] Thus, the question of shape and morphology of single-chain folded nanoassemblies of such type of amphiphilic copolymers is relevant for both experimental and theoretical viewpoints.

\subsection{Molecular dynamics}

To achieve a better understanding of the folding of PEGMA-co-FA copolymers, MD simulations were performed for four different random copolymer models (RA-RD), one blocky copolymer model (EQB), and one PEGMA homopolymer (all-HYD). All simulations were performed in water starting from outstretched random structures. To investigate the role of the solvent on the folding, we performed additional simulations in chloroform starting from the four initial structures used in the water simulations. The front views of final structures (200 ns simulation) in water and chloroform are reported in Figure 5 for RA-RD, along with those for EQB and all-HYD; top and side views of final structures in water are also shown in Figure S4.

All simulations in water reached a much more compact structure than the initial one; however, a large structural variability was also found likely due to the different distributions of PEGMA and FA counits along the main chain of each copolymer leading to different structural constraints. In all random simulations RA-RD, the copolymer finally reached a prolate globular structure, namely elongated in one direction. The fluorinated side chains FA were predominately confined in a inner hydrophobic core. In the simulations for EQB and all-HYD, instead, the final structures were more disk-shaped (Figure 5 and S4). Compared to what was found in water, the simulations in chloroform reached much more open conformations, where both different side chains remained exposed to the solvent, and fewer contacts were formed among the counits (Figure 5).

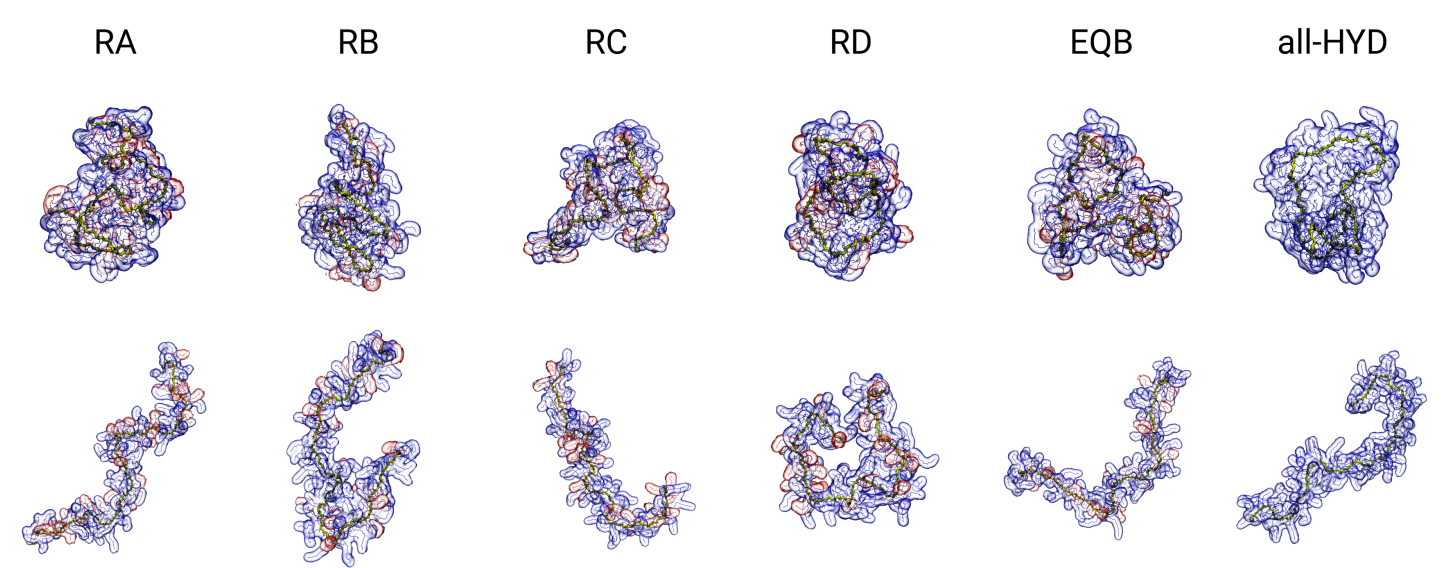

Fig. 5. Final structures ( $200 \mathrm{~ns}$, front views) from the simulations for the random copolymers RA-RD, blocky copolymer EQB and PEGMA homopolymer all-HYD in water (top row) and in chloroform (bottom row). The yellow trace represents the carbon polymer backbone; red and blue colors refer to FA and PEGMA counits, respectively.

A more quantitative description of the folding process was obtained by following the radius of gyration $R_{\mathrm{g}}$ along each simulation (Figure 6). In all simulations in water (including the blocky copolymer and the homopolymer), $R_{\mathrm{g}}$ decreased in time, whereas in chloroform, $R_{\mathrm{g}}$ oscillated around the initial value, slightly decreasing in simulation RD only. Therefore, the copolymer does not tightly fold in chloroform, rather it adopts various open conformations. Although $R_{\mathrm{g}}$ decreased along all simulations in water, the time evolution varied due to the different polymer sequence and starting conformation. Nonetheless, all simulations in water reached a value of $R_{\mathrm{g}}$ around $20-25 \AA$ within less than $200 \mathrm{~ns}$ of simulation. On the other hand, the value of $R_{\mathrm{g}}$ in chloroform was higher (30-40 ̊). Quite comparable 
values of $R_{\mathrm{g}}$ were previously found for analogue copolymers PEGMA90-co-FA10 and PEGMA70-co-FA30 in $\mathrm{D}_{2} \mathrm{O}$ solutions by small angle neutron scattering (SANS) measurements [48] (see below).
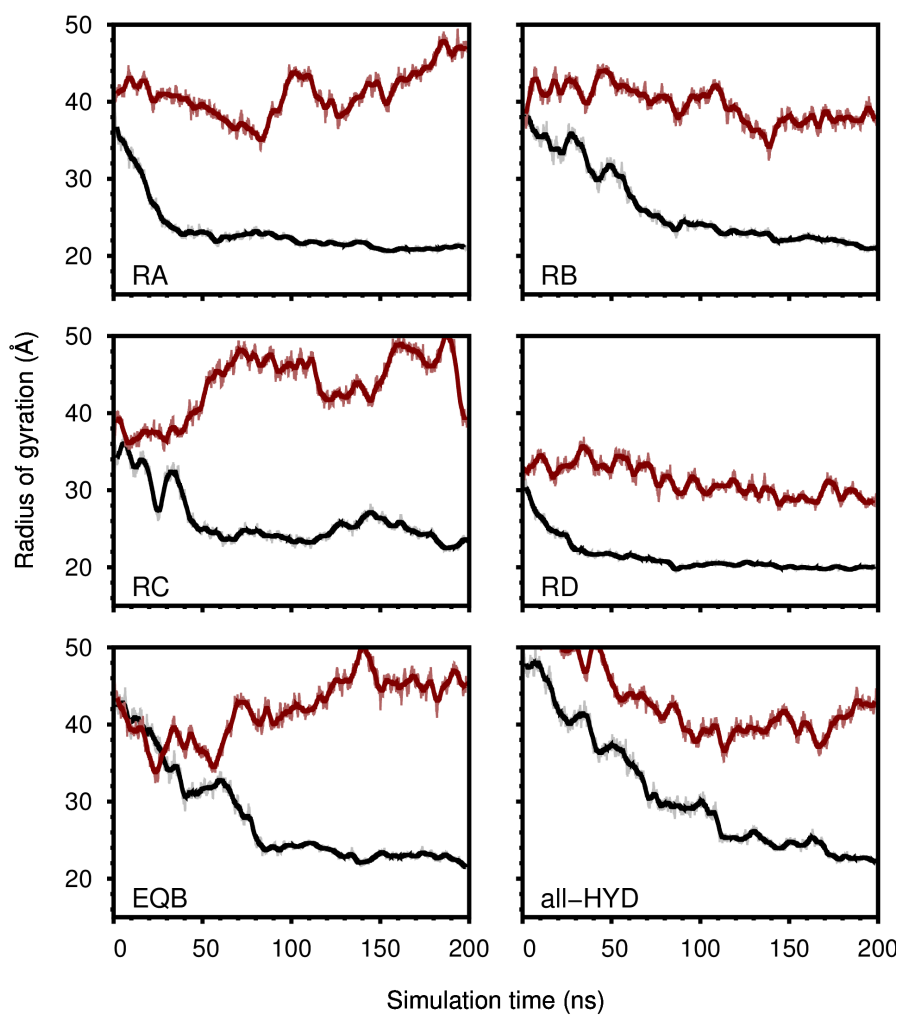

Fig. 6. Radius of gyration calculated on heavy atoms along the various simulations. Black lines represent simulations in water, dark red lines represent simulations in chloroform. All data are smoothed with a moving average over 200 frames. Thin lines represent the original non-averaged values.

The formation of globular structures in water simulations was confirmed by the analysis of shape descriptors, asphericity $\alpha$ and prolateness $p$ (Figure S5), and solvent-accessible surface area SASA (Figure 7). The values of $\alpha$ decreased, closely following the trend in $R_{\mathrm{g}}$ values, indicating that the polymer becomes more spherical as it folds. The respective $p$ indicated that three of the four random copolymer models present a definite prolate shape, with $p>0.5$ (Figure S5). In parallel, for all water simulations, SASA decreased in time (Figure 7 left), showing that the polymer structure becomes more compact, exposing less of its surface to the solvent, as a consequence of folding. By contrast, no significant changes were observed in the shape descriptors or SASA for any of the simulations in chloroform (Figures 7 left and S5), meaning that the copolymer maintains an outstretched conformation, similar to the initial structure.

In order to understand whether the two different side chains are equally exposed to solvent, we evaluated the contributions to SASA coming from PEGMA and FA units as a function of time, $X_{\mathrm{PEGMA} / \mathrm{FA}}(t)$ (Figure 7 right). In all simulations, the FA contribution was always smaller than the PEGMA contribution because FA units are shorter than PEGMA units, and thus contribute less to the total surface. However, upon folding in water the relative FA contribution to the SASA decreased much more than the PEGMA one (Figure 7 right). Therefore, the FA units become more buried in a inner region of the nanostructure, as the copolymer chain folds. On the contrary, in the simulations in chloroform the contributions of both PEGMA and FA units to the SASA were virtually constant, indicating that their different exposure to the solvent remains unchanged along the entire trajectory. Taken together, the results from the MD simulations confirm strongly different behaviors of the polymers in water and chloroform. In water solutions all 
model random copolymers self-fold into prolate globular nanostructures; by contrast more open and loose nanostructures are formed in chloroform. As expected of hydrophobic interactions, the reduction in the solventaccessible surface which accompanies the chain folding is more pronounced for the FA units, which become more buried in the core of the globule. However, a clear leading role of the hydrophobic interactions is not evident as is also highlighted by the similarities found in the investigated shape descriptors between the copolymers and the PEGMA homopolymer (all-HYD). Particularly, the simulations suggest the importance of the interplay between hydrophobic interactions and the specificity of the structural constraints of each random copolymer which results in the observed heterogeneity.
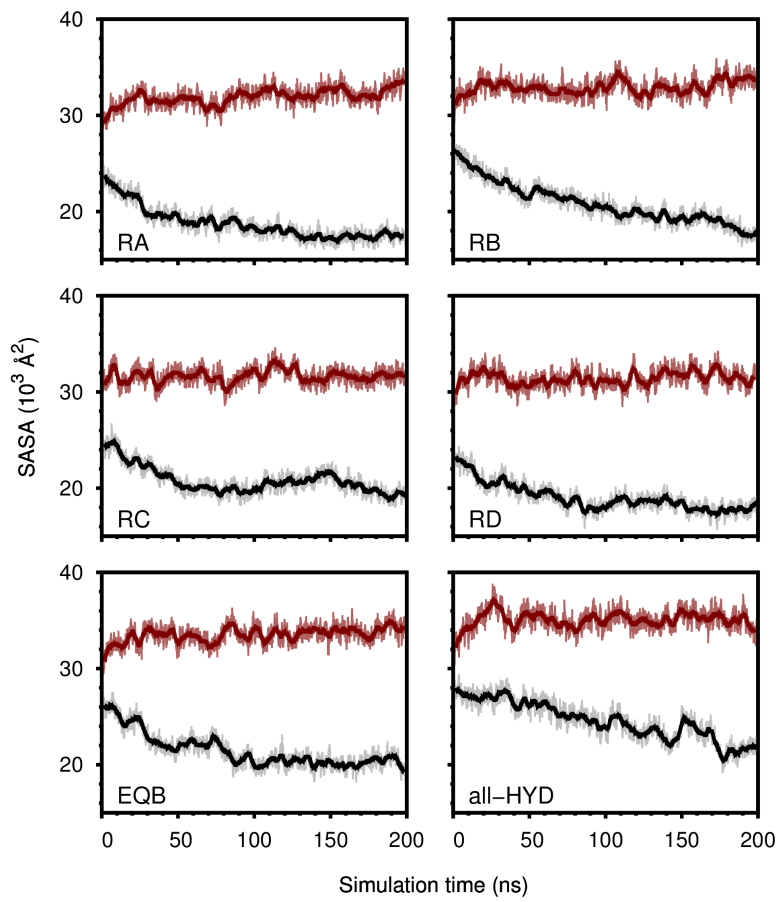

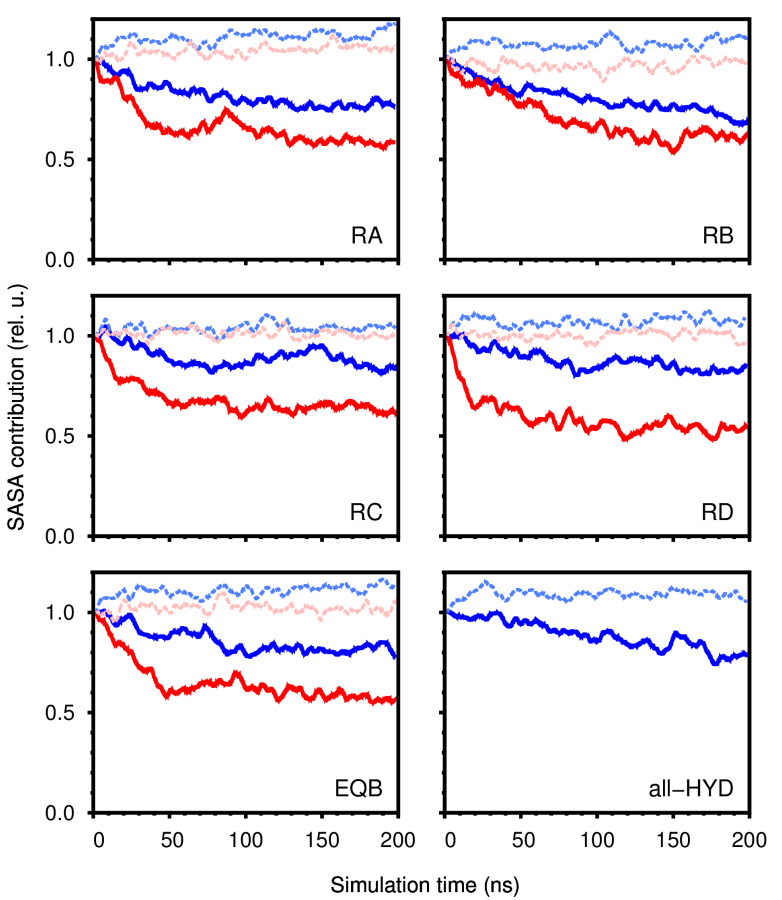

Fig. 7. Left: Solvent-accessible surface area $(S A S A)$ along the various simulations; black lines represent simulations in water, dark red lines represent simulations in chloroform (all data are smoothed with a moving average over 200 frames). Right: $X_{P E G M A / F A}(t)$ fraction of the SASA corresponding to the PEGMA (blue lines) and FA components (red lines) along the MD trajectories (see Eq.(3)); solid lines represent simulations in water, thin dashed lines represent simulations in chloroform.

In a previous work, analogue random copolymers PEGMA90-co-FA10 and PEGMA70-co-FA30, that is slightly richer and slightly poorer in PEGMA counits than the present copolymer, 90 and 70 mol\% respectively, were investigated by SANS measurements. Both copolymers formed single-chain folded nanoassemblies as prolate spheroids (in $\mathrm{D}_{2} \mathrm{O}, 2-5 \mathrm{mg} \mathrm{mL}^{-1}$, at room temperature). However, while the former exhibited a radius of gyration of $3.7 \pm 0.2 \mathrm{~nm}$ and polar axes of $9.2 \pm 0.1 \mathrm{~nm}$ and equatorial axes of $1.7 \pm 0.1 \mathrm{~nm}$ (ratio $\sim 5: 1$ ), the latter exhibited a radius of gyration of $3.4 \pm 0.2 \mathrm{~nm}$ and polar axes of $6.30 \pm 0.06 \mathrm{~nm}$ and equatorial axes of $2.77 \pm 0.03 \mathrm{~nm}$ (ratio 2:1).[48] Thus, the more hydrophilic copolymer formed more expanded prolate nanoassemblies. Based on those SANS measurements, the random copolymer PEGMA77-Co-FA23 appears to adopt a similarly prolate nanostructure, as is also expected by the atomistic MD simulations. 


\section{Conclusions}

The experimental/computational findings support the self-folding of amphiphilic random copolymer PEGMA77-co-FA23 into single-chain nanoassemblies in water. Our MD simulations show that self-folding of the copolymer in water leads to prolate globular nanostructures with a structural variability. The single-chain folding is accompanied by a reduction in the solvent-accessible surface area especially for the hydrophobic units that become more buried in the core of the globule. Hydrophobic interactions thus represent the driving force of the copolymer collapse. However, these interactions do not determine a definite arrangement of the hydrophobic units, as the structural constraints will allow different arrangements in each random copolymer sequence.

\section{Conflicts of interest}

There are no conflicts to declare.

\section{Acknowledgements}

Work performed with partial financial support from the University of Pisa (fondi Progetti di Ricerca di Ateneo, PRA_2017_17). We thank Tarita Biver for assistance with fluorescence emission studies of EtBr and Barbara Immirzi and Giovanni Dal Poggetto with SEC evaluation of molecular weights.

\section{References}

[1] J.A. Pomposo (Ed.), Single-chain polymer nanoparticles: Synthesis, characterization, simulations, and applications, Wiley-VCH, Weinheim 2017.

[2] M. Ouchi, N. Badi, J.-F. Lutz, M. Sawamoto, Single-chain technology using discrete synthetic macromolecules, Nat. Chem. 3 (2011) 917-24. doi:10.1038/nchem.1175.

[3] X. Fan, Z. Li, X.J. Loh, Recent development of unimolecular micelles as functional materials and applications, Polym. Chem. 7 (2016) 5898-5919. doi:10.1039/C6PY01006G.

[4] S. Mavila, O. Eivgi, I. Berkovich, N.G. Lemcoff, Intramolecular cross-linking methodologies for the synthesis of polymer nanoparticles, Chem. Rev. 116 (2016) 878-961. doi:10.1021/acs.chemrev.5b00290.

[5] L. Li, K. Raghupathi, C. Song, P. Prasad, S. Thayumanavan, Self-assembly of random copolymers, Chem. Commun. 50 (2014) 13417-13432. doi:10.1039/C4CC03688C.

[6] Y. Morishima, S. Nomura, T. Ikeda, M. Seki, M. Kamachi, Characterization of unimolecular micelles of random copolymers of sodium 2-(acrylamido)-2-methylpropanesulfonate and methacrylamides bearing bulky hydrophobic substituents, Macromolecules. 28 (1995) 2874-2881. doi:10.1021/ma00112a037.

[7] C.J. Riddles, W. Zhao, H.-J. Hu, M. Chen, M.R. Van De Mark, Self-assembly of water insoluble polymers into colloidal unimolecular polymer (CUP) particles of 3-9 nm, Polymer (Guildf). 55 (2014) 48-57. doi:10.1016/j.polymer.2013.11.014.

[8] G.M. Ter Huurne, M.A.J. Gillissen, A.R.A. Palmans, I.K. Voets, E.W. Meijer, The coil-to-globule transition of single-chain polymeric nanoparticles with a chiral internal secondary structure, Macromolecules. 48 (2015) 3949-3956. doi:10.1021/acs.macromol.5b00604.

[9] M. Chen, S.P. Jensen, M.R. Hill, G. Moore, Z. He, B.S. Sumerlin, Synthesis of amphiphilic polysuccinimide star 
copolymers for responsive delivery in plants, Chem. Commun. 51 (2015) 9694-9697. doi:10.1039/C5CC02726H.

[10] C.K. Lyon, A. Prasher, A.M. Hanlon, B.T. Tuten, C.A. Tooley, P.G. Frank, E.B. Berda, A brief user's guide to single-chain nanoparticles, Polym. Chem. 6 (2015) 181-197. doi:10.1039/C4PY01217H.

[11] M. Huo, N. Wang, T. Fang, M. Sun, Y. Wei, J. Yuan, Single-chain polymer nanoparticles: Mimic the proteins, Polym. (United Kingdom). 66 (2015) A11-A21. doi:10.1016/j.polymer.2015.04.011.

[12] O. Altintas, C. Barner-Kowollik, Single chain folding of synthetic polymers by covalent and non-covalent interactions: current status and future perspectives, Macromol. Rapid Commun. 33 (2012) 958-971. doi:10.1002/marc.201200049.

[13] O. Altintas, C. Barner-Kowollik, Single-chain folding of synthetic polymers: a critical update, Macromol. Rapid Commun. 37 (2016) 29-46. doi:10.1002/marc.201500547.

[14] M. Gonzalez-Burgos, A. Latorre-Sanchez, J.A. Pomposo, Advances in single chain technology, Chem. Soc. Rev. 44 (2015) 6122-6142. doi:10.1039/C5CS00209E.

[15] T. Terashima, T. Sugita, K. Fukae, M. Sawamoto, Synthesis and single-chain folding of amphiphilic random copolymers in water, Macromolecules. 47 (2014) 589-600. doi:10.1021/ma402355v.

[16] Y. Koda, T. Terashima, M. Sawamoto, H.D. Maynard, Amphiphilic/fluorous random copolymers as a new class of non-cytotoxic polymeric materials for protein conjugation, Polym. Chem. 6 (2015) $240-247$. doi:10.1039/C4PY01346H.

[17] Y. Koda, T. Terashima, M. Sawamoto, Multimode self-folding polymers via reversible and thermoresponsive self-assembly of amphiphilic/fluorous random copolymers, Macromolecules. 49 (2016) 4534-4543. doi:10.1021/acs.macromol.6b00998.

[18] E. Guazzelli, E. Masotti, T. Biver, A. Pucci, E. Martinelli, G. Galli, The self-assembly over nano- to submicrolength scales in water of a fluorescent julolidine-labeled amphiphilic random terpolymer, J. Polym. Sci. Part A Polym. Chem. 56 (2018) 797-804. doi:10.1002/pola.28955.

[19] E. Martinelli, L. Annunziata, E. Guazzelli, A. Pucci, T. Biver, G. Galli, The temperature-responsive nanoassemblies of amphiphilic random copolymers carrying poly(siloxane) and poly(oxyethylene) pendant chains, Macromol. Chem. Phys. (2018) 1800082. doi:10.1002/macp.201800082.

[20] E. Martinelli, I. Del Moro, G. Galli, M. Barbaglia, C. Bibbiani, E. Mennillo, M. Oliva, C. Pretti, D. Antonioli, M. Laus, Photopolymerized network polysiloxane films with dangling hydrophilic/hydrophobic chains for the biofouling release of invasive marine serpulid Ficopomatus enigmaticus, ACS Appl. Mater. Interfaces. 7 (2015) 8293-8301. doi:10.1021/acsami.5b01522.

[21] Y.Y. Durmaz, E.L. Sahkulubey, Y. Yagci, E. Martinelli, G. Galli, A novel poly( p -phenylene) containing alternating poly(perfluorooctylethyl acrylate-co-methyl methacrylate) and polystyrene grafts by combination of atom transfer radical polymerization and Suzuki coupling processes, J. Polym. Sci. Part A Polym. Chem. 50 (2012) 4911-4919. doi:10.1002/pola.26320.

[22] E. Martinelli, G. Galli, S. Krishnan, M.Y. Paik, C.K. Ober, D.A. Fischer, New poly(dimethylsiloxane)/poly(perfluorooctylethyl acrylate) block copolymers: structure and order across multiple length scales in thin films, J. Mater. Chem. 21 (2011) 15357. doi:10.1039/c1jm12044a.

[23] E. Martinelli, D. Gunes, B.M. Wenning, C.K. Ober, J.A. Finlay, M.E. Callow, J.A. Callow, A. Di Fino, A.S. Clare, G. 
Galli, Effects of surface-active block copolymers with oxyethylene and fluoroalkyl side chains on the antifouling performance of silicone-based films, Biofouling. $32 \quad$ (2016) 81-93. doi:10.1080/08927014.2015.1131822.

[24] E. Martinelli, A. Glisenti, B. Gallot, G. Galli, Surface properties of mesophase-forming fluorinated bicycloacrylate/polysiloxane methacrylate copolymers, Macromol. Chem. Phys. 210 (2009) 1746-1753. doi:10.1002/macp.200900225.

[25] Y. Kimura, T. Terashima, M. Sawamoto, Self-assembly of amphiphilic random copolyacrylamides into uniform and necklace micelles in water, Macromol. Chem. Phys. 218 (2017) 1-11. doi:10.1002/macp.201700230.

[26] J.-F. Lutz, Polymerization of oligo(ethylene glycol) (meth)acrylates: Toward new generations of smart biocompatible materials, J. Polym. Sci. Part A Polym. Chem. 46 (2008) 3459-3470. doi:10.1002/pola.22706.

[27] B. Trzebicka, D. Szweda, S. Rangelov, A. Kowalczuk, B. Mendrek, A. Utrata-Wesołek, A. Dworak, (Co)polymers of oligo(ethylene glycol) methacrylates-temperature-induced aggregation in aqueous solution, J. Polym. Sci. Part A Polym. Chem. 51 (2013) 614-623. doi:10.1002/pola.26410.

[28] D. Szweda, R. Szweda, A. Dworak, B. Trzebicka, Thermoresponsive poly[oligo(ethylene glycol) methacrylate]s and their bioconjugates - Synthesis and solution behavior, Polimery/Polymers. 62 (2017) 298-310. doi:10.14314/polimery.2017.298.

[29] J.A. Pomposo, I. Perez-Baena, F. Lo Verso, A.J. Moreno, A. Arbe, J. Colmenero, How far are single-chain polymer nanoparticles in solution from the globular state?, ACS Macro Lett. (2014) 767-772. doi:10.1021/mz500354q.

[30] J.A. Pomposo, J. Rubio-Cervilla, A.J. Moreno, F. Lo Verso, P. Bacova, A. Arbe, J. Colmenero, Folding single chains to single-chain nanoparticles via reversible interactions: what size reduction can one expect? Macromolecules. 50 (2017) 1732-1739. doi:10.1021/acs.macromol.6b02427.

[31] A.J. Moreno, F. Lo Verso, A. Sanchez-Sanchez, A. Arbe, J. Colmenero, J.A. Pomposo, Advantages of orthogonal folding of single polymer chains to soft nanoparticles, Macromolecules. 46 (2013) 9748-9759. doi:10.1021/ma4021399.

[32] F. Lo Verso, J.A. Pomposo, J. Colmenero, A.J. Moreno, Multi-orthogonal folding of single polymer chains into soft nanoparticles, Soft Matter. 10 (2014) 4813-21. doi:10.1039/c4sm00459k.

[33] A. Arbe, J.A. Pomposo, A.J. Moreno, F. LoVerso, M. González-Burgos, I. Asenjo-Sanz, A. Iturrospe, A. Radulescu, O. Ivanova, J. Colmenero, Structure and dynamics of single-chain nano-particles in solution, Polym. (United Kingdom). 105 (2016) 532-544. doi:10.1016/j.polymer.2016.07.059.

[34] P.J.M. Stals, M.A.J. Gillissen, T.F.E. Paffen, T.F.A. de Greef, P. Lindner, E.W. Meijer, A.R.A. Palmans, I.K. Voets, Folding polymers with pendant hydrogen bonding motifs in water: the effect of polymer length and concentration on the shape and size of single-chain polymeric nanoparticles, Macromolecules. 47 (2014) 2947-2954. doi:10.1021/ma500273g.

[35] F. Lo Verso, J.A. Pomposo, J. Colmenero, A.J. Moreno, Simulation guided design of globular single-chain nanoparticles by tuning the solvent quality, Soft Matter. 11 (2015) 1369-1375. doi:10.1039/C4SM02475C.

[36] H. Rabbel, P. Breier, J.-U. Sommer, Swelling behavior of single-chain polymer nanoparticles: theory and simulation, Macromolecules. 50 (2017) 7410-7418. doi:10.1021/acs.macromol.7b01379.

[37] M.J. Waring, Complex formation between ethidium bromide and nucleic acids, J. Mol. Biol. 13 (1965) $269-282$. 
doi:10.1016/S0022-2836(65)80096-1.

[38] J. Wang, R.M. Wolf, J.W. Caldwell, P.A. Kollman, D.A. Case, Development and testing of a general amber force field, J. Comput. Chem. 25 (2004) 1157-1174. doi:10.1002/jcc.20035.

[39] C.I. Bayly, P. Cieplak, W. Cornell, P.A. Kollman, A well-behaved electrostatic potential based method using charge restraints for deriving atomic charges: the RESP model, J. Phys. Chem. 97 (1993) 10269-10280. doi:10.1021/j100142a004.

[40] W.L. Jorgensen, J. Chandrasekhar, J.D. Madura, R.W. Impey, M.L. Klein, Comparison of simple potential functions for simulating liquid water, J. Chem. Phys. 79 (1983) 926-935. doi:10.1063/1.445869.

[41] T. Fox, P.A. Kollman, Application of the RESP methodology in the parametrization of organic solvents, J. Phys. Chem. B 102 (1998) 8070-8079. doi:10.1021/jp9717655.

[42] D.A. Case, V. Babin, J.T. Berryman, R.M. Betz, Q. Cai, D.S. Cerutti, T.E. Cheatham III, T.A. Darden, R.E. Duke, H. Gohlke, A.W. Goetz, S. Gusarov, N. Homeyer, P. Janowski, J. Kaus, I. Kolossváry, A. Kovalenko, T.S. Lee, S. LeGrand, T. Luchko, R. Luo, B. Madej, K.M. Merz, F. Paesani, D.R. Roe, A. Roitberg, C. Sagui, R. Salomon-Ferrer, G. Seabra, C.L. Simmerling, W. Smith, J. Swails, R.C. Walker, J. Wang, R.M. Wolf, X. Kollman, P.A. Wu, Amber 14, University of California, San Francisco, 2014. doi:10.1007/s13398-014-0173-7.2.

[43] M. Connolly, Solvent-accessible surfaces of proteins and nucleic acids, Science 221 (1983) 709-713. doi:10.1126/science.6879170.

[44] A. Varshney, F.P. Brooks, W.V. Wright, Computing smooth molecular surfaces, IEEE Comput. Graph. Appl. 14 (1994) 19-25. doi:10.1109/38.310720.

[45] N.H. Nguyen, J. Kulis, H. Sun, Z. Jia, B. Van Beusekom, M.E. Levere, D.A. Wilson, J. Monteiro, V. Percec, A comparative study of the SET-LRP of oligo(ethylene oxide) methyl ether acrylate in DMSO and in H2O, Polym. Chem. 2 (2013) 144-155. doi:10.1039/c2py20782f.

[46] N.H. Nguyen, X. Leng, H.J. Sun, V. Percec, Single-electron transfer-living radical polymerization of oligo(ethylene oxide) methyl ether methacrylate in the absence and presence of air, J. Polym. Sci. Part A Polym. Chem. 51 (2013) 3110-3122. doi:10.1002/pola.26718.

[47] J.R. Lakowicz, G. Weber, Quenching of protein fluorescence by oxygen. Detection of structural fluctuations in proteins on the nanosecond time scale, Biochemistry. 12 (1973) 4171-4179. doi:10.1021/bi00745a021.

[48] E. Martinelli, E. Guazzelli, G. Galli, M.T.F. Telling, G.D. Poggetto, B. Immirzi, F. Domenici, G. Paradossi, Prolate and temperature-responsive self-assemblies of amphiphilic random copolymers with perfluoroalkyl and polyoxyethylene side chains in solution, Macromol. Chem. Phys. 219 (2018) 1800210. doi:10.1002/macp.201800210. 